\title{
GLUTATHIONE AND MORBIDITY IN A COMMUNITY-BASED SAMPLE OF ELDERLY
}

\author{
Mara Julius, ${ }^{*}$ Calvin A. Lang, ${ }^{2}$ Lillian Gleiberman, ${ }^{1}$ Ernest Harburg, ${ }^{1,3}$ \\ WAYNE DIFRANCEISCO' ${ }^{1}$ and ANTHONY SCHORK ${ }^{4}$ \\ 'Department of Epidemiology, School of Public Health, University of Michigan, Ann Arbor, MI \\ 48109 , ${ }^{2}$ Department of Biochemistry, School of Medicine, University of Louisville, Louisville, KY \\ 40292, ${ }^{3}$ Department of Psychology, School of Literature, Science and the Arts, University of \\ Michigan, MI 48109 and ${ }^{4}$ Department of Biostatistics, School of Public Health, University of \\ Michigan, MI 48109, U.S.A.
}

(Received in revised form 2 February 1994)

\begin{abstract}
This study examined the association of blood glutathione level, a potential marker of physiological/functional aging, with a number of biomedical/psychological traits in a subgroup $(N=33)$ of a representative sample of community-based elderly. Higher glutathione levels were associated with fewer number of illnesses $(p<0.05)$, higher levels of self-rated health $(p<0.01)$, lower cholesterol $(p<0.05)$, lower body mass index, and lower blood pressures. Subjects with diagnoses of arthritis, diabetes, or heart disease (as assessed by physicians) had at least marginally significant lower glutathione levels than those who were disease free. Glutathione, together with age and a measure of suppressed anger, accounted for $39 \%$ of the variance of an index of morbidity. Glutathione, by itself, accounted for $24 \%$ of the variance. To our knowledge, this is the first evidence of an association of higher glutathione levels with higher levels of physical health in a sample of community-based elderly. Further studies in large samples are needed to investigate glutathione as a potential overall health risk factor for morbidity among the elderly.
\end{abstract}

Glutathione Chronological age Suppressed anger Morbidity Aging

\section{INTRODUCTION}

The concept of physiological or functional age arose out of the observations that during senescence, elderly people of the same chronological age manifest different levels of decline for numerous physiological parameters. Among several approaches to understanding physiological decline in later life, the search for a metabolite, easily and objectively measured, which would be a marker of functional age, has assumed a central role [1]. Recently, the tripeptide glutathione

*All correspondence should be addressed to: Dr M. Julius, Department of Epidemiology, University of Michigan, 109 Observatory St, Ann Arbor, MI 48109-2029, U.S.A.
(GSH) has been suggested as a prime candidate [2].

Glutathione has several major physiological functions: (1) protecting cells against destructive effects of reactive oxygen intermediates and free radicals; (2) detoxifying external substances such as drugs and environmental pollutants; (3) maintaining red cell membrane stability; and (4) enhancing immunological function through its effects on lymphocytes $[2,3]$. These widespread functions suggest that the level of glutathione may have major health effects on the molecular, cellular and organ levels of individuals.

Initial work using animal models of mosquito, mouse and rat, indicated that gluta- 
thione declined with older age, and paralleled the survival curves [4-6]; correction of a glutathione deficiency enhanced longevity in the mosquito [7]. Following these observations, investigators began to examine glutathione in human samples. In a convenience sample of clinically healthy groups, glutathione levels were significantly lower in old (60-79 years) when compared to mature (40-59 years) subjects. However, levels in the very old (80-99 years) were similar to those of the mature group. This curvilinear pattern suggests that this sclected sample of very old healthy men and women may represent survivors with extreme longevity [8]. In another convenience sample of elderly white women, selected on the basis of good health, blood glutathione levels were found to be the highest ever observed [9]. The purpose of the current study is to examine glutathione, for the first time, in a subgroup of a random sample of community-based elderly with wider health range (from poor to excellent). The general hypothesis of the research is that higher glutathione levels are associated with better overall health.

\section{SUBJECTS AND METHODS}

Subjects for this pilot study were selected from a random sample $(N=172)$ of urban, white, independently living elderly over the age of 60 , interviewed and medically examined in 1984. The design for selecting the sample for this pilot study $(N=33)$ aimed to have approximately equal numbers of individuals who rated their health low, medium, and high. We also wanted approximately equal numbers of males and females in two age groups: 60-69 years and $\geqslant 70$ years. For those aged $60-69$, respondents were first stratified by low, medium, and high self-rated health, and then by gender. Thirteen respondents were randomly selected from this group. All 20 survivors over the age of 70 were then included in this pilot sample. In 1986, the subgroup $(N=33)$ was reinterviewed, and blood samples were drawn for the glutathione assay. All data for this study were derived from questionnaires and medical examinations administered in 1984 and 1986 at Providence Hospital in Southfield, Michigan.

\section{Health measures}

In 1986, using a double blind method, glutathione levels were determined in fasting blood samples. Blood samples were collected in ethylenediamine tetraacetic acid-prepared tubes, immediately placed on crushed ice $\left(0^{\circ} \mathrm{C}\right)$, and then stored at $0-4^{\circ} \mathrm{C}$. Samples were shipped in dry ice to the laboratory of Dr C. A. Lang at the University of Louisville for processing. Validation of the sample processing and storage conditions was carried out prior to the beginning of this study and showed that glutathione was stable for more than 20 days in blood samples prepared in this manner [8]. Glutathione was analyzed by the HPLC-DEC method and cxpressed as $\mu \mathrm{g}$ glutathionc $/ 10^{10}$ erythrocytes. This method simultaneously measures reduced and oxidized glutathione, cysteine and other thiols [10].

All other health measures were determined in 1984 during an extensive medical examination at Providence Hospital in Southfield, Michigan. Blood pressure was measured by the examining physician on the right arm, with respondent seated, and before other medical tests were performed. Cholesterol was assayed in fasting blood samples. Body mass index was calculated by dividing weight (in $\mathrm{kg}$ ) by height (in $\mathrm{m}^{2}$ ).

Based on the physician's assessment of presence/absence of a series of illnesses or medical conditions, as well as review of more objective tests (e.g. EKG for heart disease, blood sugar levels for diabetes), a simple summary measure of number of illnesses constituted the mobidity index.

Subjects also responded to a self-rated question, "How would you rate your overall health at the present time?" A dichotomous index of self-perceived health divided the sample into those who responded "good" or "excellent" versus those who rated their health as "very poor," "poor," or "fair." Idler et al. [11] have demonstrated that global self-evaluated health status is a significant independent predictor of mortality and have reviewed other studies indicating the same result. Subjects were also asked whether or not they smoked cigarettes or drank alcoholic beverages.

\section{Suppressed anger measure}

Suppressed anger has previously been shown to be related to blood pressure $[12,13]$ and to cardiovascular morbidity [14], and mortality [15]. A measure of anger suppression modified from prior scales $[13,15]$ was computed as the mean score of three items, namely, "boiling inside ...," "brooding ...," and "feeling hurt ...," (but not showing anger), relating to a respondent's characteristic reactions to anger- 
provoking situations. Subjects who were low on suppressed anger had a mean score of "1" and those who "always" suppressed anger had a mean score of " 4 ".

\section{Statistical analyses}

Statistical analyses consisted of determining first order and partial (on age) correlations; analyses of covariance (using age as a covariate); and simple and multiple regression modeling. Because of the pilot character of these analyses (we are assessing possible correlations between glutathione and health indicators), we have used a graduated declaration of significance: if $p<0.10$, we have labeled the finding as marginally significant; and if $p<0.05$, we have labeled the finding as statistically significant. If $p<0.01$ or $p<0.001$, further differentiation of this level of statistical significance is noted.

\section{RESULTS}

We first examined (Table 1) the relationship of a number of morbidity measures to glutathione using simple correlations. Glutathione was found to be positively associated with age $(p<0.01)$ and marginally positively associated with alcohol status $(p<0.10)$, and negatively associated with the morbidity index $(p<0.01)$ and body mass index $(p<0.05)$. Two other predictors of morbidity, cholesterol and systolic blood pressure, were negatively associated with glutathione, as expected, but did not reach significance.

The strong positive association between age and glutathione suggested that age might be a potentially confounding influence on the re-

Table 1. Correlations of glutathione (GSH) with measures of morbidity among Southfield elderly $(n=33)$

\begin{tabular}{lcc}
\hline & $\begin{array}{c}\text { First-order } \\
\text { correlation } \\
\text { coefficient }\end{array}$ & $\begin{array}{c}\text { Partial } \\
\text { correlation } \\
\text { coefficient }\end{array}$ \\
\hline Age & $0.449^{* * *}$ & $\mathrm{n} / \mathrm{a}$ \\
Morbidity index (sum) & $-0.495^{* * *}$ & $-0.559^{* * * *}$ \\
Suppressed anger & 0.108 & 0.026 \\
Body mass index & $-0.324^{* *}$ & -0.226 \\
Cholesterol & -0.158 & -0.004 \\
Systolic blood pressure & -0.206 & -0.208 \\
Alcohol status (no/yes) & $0.300^{*}$ & $0.270^{*}$ \\
Cigarette smoking & 0.090 & 0.120 \\
(no/yes) & & \\
\hline
\end{tabular}

†Correlation coefficients for the continuous variables are Pearson $R$ s. For alcohol status and cigarette smoking the correlation measures used are etas.

†Correlations cited are for each measure with GSH, controlling for age.

Significance level: ${ }^{*} p<0.10 ;{ }^{* *} p<0.05 ;{ }^{* * *} p<0.01$; ${ }^{* * * * *} p<0.001$. lationship between glutathione and the other health attributes; glutathione was thus adjusted for age. Partial correlations, using age-adjusted glutathione increased the significance of the morbidity index $(p<0.001)$; however, body mass index was no longer significant.

We next (Table 2) examined the relationship between age-adjusted glutathione levels and the physician's and subjects' ascertained health measures, using analysis of variance. Glutathione levels were analyzed for several of the individual illnesses. An illness was selected for separate analysis only if at least four respondents had the condition. For every illness, except urinary tract infections, those subjects who possessed the condition had a lower mean glutathione level than those without the condition. Two of the associations were marginally significant: arthritis and diabetes $(p<0.10)$, and a third, heart disease, was statistically significant $(p<0.05)$. In addition, respondents who rated their health as "good" or "excellent" had significantly $(p<0.01)$ higher blood levels of glutathione than did their counterparts in the "very poor"/"poor"/"fair" group.

Finally (Table 3), separate regressions of individual variables on the morbidity index indicated significant associations for 3 of 8 variables (column 1): glutathione, body mass index and alcohol use. The $r^{2}$ for these variables, respectively, were $0.24,0.11$, and 0.09 . The multiple regression model, including all the predictors (column 2) accounted for $39 \%$ of the variance in the morbidity measure and was significant $(p=0.003)$. Glutathione was the strongest of partial relationships $(\beta=-0.64)$. The negative coefficient indicates that higher glutathione levels were associated with lower morbidity scores. Although the correlation between age and morbidity in the simple regression was not significant, the stepwise regression indicated that age, adjusted for all other covariates, was a marginally significant independent predictor $(p<0.10)$, as was the measure of suppressed anger.

\section{DISCUSSION}

This study provided evidence that glutathione, together with age, a measurc of suppressed anger, and other predictors, accounted for $39 \%$ of the variance of an index of morbidity in a sample of independently living elderly adults. However, glutathione when examined by itself accounted by far for the largest portion of 
Table 2. Mean glutathione (GSH) by measures of morbidity among Southfield elderly $(n=33)$

\begin{tabular}{lrccc}
\hline Measure & $(n)$ & $\begin{array}{c}\text { Unadjusted } \\
\text { Mean GSH }\end{array}$ & $\begin{array}{c}\text { Age-adjusted } \\
\text { Mean GSH }\end{array}$ & $F$-value $\dagger$ \\
\hline Morbidity Index (1984) [2] & & & & \\
$\quad$ O-1 illnesses & $(7)$ & 454.8 & 477.3 & $5.29^{* *}$ \\
$\quad$ or more illnesses & $(26)$ & 399.5 & 393.5 & \\
Arthritis & $(13)$ & 446.6 & 448.3 & $3.91^{*}$ \\
$\quad$ No & $(19)$ & 388.1 & 386.9 & \\
$\quad$ Yes & & & & \\
High blood pressure & $(22)$ & 426.4 & 425.8 & 1.80 \\
$\quad$ No & $(11)$ & 381.0 & 382.3 & \\
$\quad$ Yes & $(27)$ & 430.5 & 430.5 & $8.68^{* * *}$ \\
Heart disease & $(6)$ & 324.9 & 324.7 & \\
$\quad$ No & & & & \\
$\quad$ Yes & $(27)$ & 420.1 & 419.9 & 1.43 \\
Circulatory symptoms & $(6)$ & 371.7 & 372.3 & \\
$\quad$ No & & & & \\
$\quad$ Yes & $(29)$ & 422.2 & 421.6 & $3.45^{*}$ \\
Diabetes & $(4)$ & 332.1 & 336.7 & \\
$\quad$ No & & & & \\
$\quad$ Yes & $(26)$ & 418.9 & 419.4 & 1.03 \\
Stomach symptoms & $(7)$ & 383.1 & 381.1 & \\
$\quad$ No & & & & \\
$\quad$ Yes & $(22)$ & 396.1 & 409.6 & 0.02 \\
Urinary tract infection & $(11)$ & 441.5 & 414.5 & \\
$\quad$ No & & & & \\
Yes & $(24)$ & 439.0 & 438.6 & $10.34^{* * *}$ \\
Self-rated health (1986) & 337.4 & 338.5 & \\
$\quad$ Good, excellent & Very poor, poor, fair & $(9)$ & & \\
\hline
\end{tabular}

$\dagger F$-value cited is for the main effect in the analysis of variance, where GSH is adjusted for age.

$\ddagger$ The index equals the sum of "yes" responses (diagnosed and/or treated for) the following conditions: arthritis, glaucoma, asthma, emphysema, TB, high blood pressure, heart disease, circulatory problems, diabetes, ulcers, stomach problems, liver disease, kidney disease, urinary tract infection, cancer/leukemia, anemia, stroke, pneumonia, chronic headaches, and up to two other conditions.

Significance level: ${ }^{*} p<0.10 ;{ }^{* *} p<0.05 ;{ }^{* *} p<0.01$.

Table 3. Multiple regression of the morbidity index on glutathione (GSH) and other health risk factors among Southfield elderly $(n=33)$

\begin{tabular}{|c|c|c|}
\hline & $\begin{array}{l}\text { Separate bivariate } \\
\text { regressions }\end{array}$ & $\begin{array}{l}\text { Multivariate } \\
\text { regression model }\end{array}$ \\
\hline & $\begin{array}{c}R \text {-Square of predictor } \\
\text { without any other } \\
\text { covariates }\end{array}$ & $\begin{array}{l}\text { Standardized } \\
\text { coefficients } \\
(\beta) \dagger\end{array}$ \\
\hline Glutathione & $0.245^{* * *}$ & $-0.637^{* * * *}$ \\
\hline Age & 0.004 & $0.299^{*}$ \\
\hline Suppressed anger & 0.047 & $0.260^{*}$ \\
\hline Body mass index & $0.113^{* *}$ & 0.225 \\
\hline $\begin{array}{l}\text { Cigarette smoking } \\
(0=\text { no; } 1=\text { yes })\end{array}$ & 0.030 & -0.156 \\
\hline $\begin{array}{l}\text { Alcohol status } \\
(0=\text { non-drinker; } 1=\text { drinker })\end{array}$ & $0.087 * *$ & -0.072 \\
\hline Cholesterol & 0.010 & -0.042 \\
\hline Systolic blood pressure & 0.059 & -0.011 \\
\hline & \multicolumn{2}{|c|}{ Full Model: $\begin{aligned} R \text {-Square }=0.39 \\
F \text {-value }=5.92(p=0.003)\end{aligned}$} \\
\hline
\end{tabular}

†When the stepwise regression model was generated, only the first three predictors (in the order listed) were found to be at least marginally significant.

Significance level: ${ }^{*} p<0.10 ;{ }^{* *} p<0.05 ;{ }^{* *} p<0.01 ;{ }^{* * * *} p<0.001$. 
the variance $(24 \%)$. To our knowledge, this is the first evidence of an association of higher glutathione with better physical health in a sample of community-based elderly.

Individuals diagnosed as having at least one of several chronic illnesses had lower glutathione levels than those who were disease free, in six of the seven comparisons, and at least marginally significant in three of seven. Elsewhere, lower glutathione levels have been found in clinical studies of patients with chronic renal failure [16], malignant disorders [17], maturity onset diabetes [18], hypertension [19], and HIV infection [20-22]. In addition, after examining a series of consecutive hospital admissions, Lang [23] found a glutathione deficiency in $77 \%$ of patients with a variety of chronic diseases.

Although in this study the relationship between suppressed anger and morbidity is marginal, it is congruent with results from studies with larger samples which relate angercoping styles to hypertension $[12,13]$, morbidity [14], and mortality [15].

In this study, mean glutathione levels increased with age. At first glance, this appears to contradict the observations in animal models that glutathione decreased with age and paralleled the survivorship curve $[4,5]$. However, in a more extensive study, Lang [8] found a curvilinear relationship between glutathione and age in humans; an increase of glutathione deficient individuals with increasing age across six decades of the adult life span was observed, but in the oldest group ( $80-99$ years), there were fewer deficient individuals $(24 \%)$ than in the $60-79$ year old group ( $46 \%)$. Lang suggests that these very old were "survivors," and that those with glutathione deficient levels may have died off. This may also be true in the present study of elderly. It would be important to examine glutathione in subsamples of elderly residing in nursing homes within a larger representative sample.

Presently, it is unclear how glutathione exerts its action on the body in the presence of a disease state. It is possible that normal glutathione stores of overweight individuals may become depleted while fighting the toxic products of lipid peroxidation. Consequently, when challenged by precursors of chronic diseases, the body may not have adequate reserves to combat a variety of noxious stimuli, resulting in the onset of disease. Glutathione can also neutralize the toxic products of medications, further depleting its stores and compromising the ability of the individual to fight attacks by other diseases or environmental pollutants.

Recent research has also indicated that glutathione supplements may attenuate or reverse certain pathophysiological states. Glutathione has been used to treat idiopathic pulmonary fibrosis [24], hepatocellular carcinoma [25], and low sperm count [26] with some positive results. Finally, under experimental conditions, correction of glutathione deficiency in aging mosquitos increased longevity $40 \%$ [7]. The evidence from small clinical studies that low levels of glutathione are associated with a number of morbid states is rapidly accumulating. Inasmuch as the glutathione measure is inexpensively and reliably assessed, testing it as a possible clinical indicator of future morbidity or immunodeficiency in diverse samples is worthwhile.

One limitation of the present study is the 2 year hiatus between the physical examination and the glutathione assays. However, in a study with a 1 year follow-up, glutathione levels remained stable for the majority of the study participants [9]. Though the procedures for selecting this subsample were random, one must be cautious about findings from any small-numbered sample. A clearer understanding of the nature and extent of glutathione's role in the aging process awaits further investigation. Both longitudinal studies of large representative samples as well as well-designed experimental studies will be required.

Acknowledgements - This research was supported in part by the City of Southfield, MI, and Providence Hospital, Southfield, MI; and the Louisville Longitudinal Longevity Program, University of Louisville, Louisville, KY.

\section{REFERENCES}

1. Shock NW. Normal Aging and Senescence: Physiological and Chronological Age. In: Dietz AA, Marcum VS, Eds. Aging-Its Chemistry. Arnold O. Beckman Third Conference in Clinical Chemistry. Colarado Springs; 1979: 3-24.

2. Lang CA. Research strategies for the study of nutrition and aging. In: Chen LH, Ed. Nutritional Aspects of Aging, Vol. 1. Boca Raton, FL: CRC Press Inc.; 1986: 3-18.

3. Meister A. Glutathione metabolism and its selective modifications. J Biol Chem 1988; 263(33): 17,205-17,208.

4. Hazelton GA, Lang CA. Glutathione biosynthesis in the aging adult yellow-fever mosquito Aedes aegypti (Louisville). Biochem J 1983; 210; 289-295.

5. Hazelton GA, Lang CA. Glutathione peroxidase and reductase activities in the aging mouse. Mech Aging Devep 1985; 29: 71-81.

6. Lang CA, Wu W, Chen TS, et al. Blood glutathione: a biochemical index of life span enhancement in the diet restricted Lobund-Wister rat. In: DL Snyder, Ed. 
The Effects of Dietary Restriction on Aging and Disease in the Germfree and Conventional Lobund-Wister Rat. New York: Alan R. Liss; 1988.

7. Richie JP Jr, Mills BJ, Lang CA. Correction of a glutathione deficiency in the aging mosquito increases its longevity. Proc Soc Exp Biol Med 1987; 184: 113-117.

8. Lang CA, Naryshkin S, Schneider DL et al. Low blood glutathione in healthy aging adults. J Lab Clin Med 1992; 120(5): 720-725.

9. Lang CA, Schneider DL, Naryshkin S. Further evidence on blood glutathione as an index of aging women. Gerontologist 1982; 22: 54 .

10. Richie JP Jr, Lang CA. The determination of glutathione, cyst(e)ine and other thiols and disulfides in biological samples using HPLC and dual electrochemical detection. Anal Biochem 1987; 163: 9-15.

11. Idler EL, Kasl SV, Lemke JH. Self-evaluated health and mortality among the elderly in New Haven, Connecticut, and Iowa and Washington Counties, Iowa, 1982-1986. Am J Epidemiol 1990; 131: 91-103.

12. Esler $\mathbf{M}$, Julius $\mathbf{S}$, Zweifler $A$ et al. Mild high-renin essential hypertension: Neurogenic human hypertension? N Engl J Med 1977; 296: 405-411.

13. Harburg E, Blakelock EH Jr, Roeper PJ. Resentful and reflective coping with arbitrary authority and blood pressure: Detroit. Psychosom Res 1979; 41: 189-202.

14. Chesney MA, Rosenman RH, Eds Anger and Hostility in Cardiovascular and Behavioral Disorders. Washington: Hemisphere Publishing Corp.; 1985: 107-147.

15. Julius $\mathrm{M}$, Harburg $\mathrm{E}$, Cottington EM et al. Angercoping types, blood pressure, and all-cause mortality: A follow-up in Tecumseh, Michigan (1971-1983). Am J Epidemivl 1986; 124: 220-233

16. Seth RK, Saini AS, Aggarwal SK. Glutathione peroxi- dase activity and reduced glutathione content in erythrocytes of patients with chronic renal failure. Scand J Haematol 1985; 35: 201-204.

17. Beutler E, Gelbert T. Plasma glutathione in health and in patients with malignant disease. $J$ Lab Clin Med 1985; 105: 581-584.

18. Awadallah R, El-Dessoukey EA, Doss $\mathrm{H}$ et al. Bloodreduced glutathione, pyruvic acid, citric acid, ceruloplasm in oxidase activity and certain mineral changes in diabetes mellitus before and after treatment. $\mathbf{Z}$ Ernährungswissenschaft 1978; 17: 72-78.

19. Murakami E, Hiwada K, Kokubu T. The role of brain glutathione in blood pressure regulation. Jap Circulation J 1988; 52: 1299-1300.

20. Eck HP, Grunder H, Hartmann W et al. Low cencentration of acid-soluble thiol (cysteine) in blood plasma of HIV-1-infected patients. Biol Chem Hoppe-Seyler 1989; 370: 101

21. Buhl R, Jaffee HA, Holroyd KJ et al. Glutathione deficiency and HIV. Lancet 1990; i: 546.

22. Staal FJT, Roederer M, Israelski DM et al. Intracellular glutathione levels in T-cell subsets decrease in HIV infected individuals. AIDS Res Hum Retroviruses 1992; 8: $305-311$.

23. Lang CA, Mills BJ, Mastropaolo W. Glutathione deficiency occurs in $77 \%$ of hospitalized subjects of various ages. Gerontologist 1990; 30: 39A.

24. Borok Z, Buhl R, Grimes GJ et al. Effect of glutathione aerosol on oxidant-antioxidant imbalance in idiopathic pulmonary fibrosis. Lancet 1991; 338: 215.

25. Dalhoff K, Ranek L, Mantoni M, Poulsen HE. Glutathione treatment of hepatocellular carcinoma. Liver 1992; 12: 341-343.

26. Lenzi A, Lombardo F, Gandini E et al. Glutathine therapy for male infertility. Arch Andrology 1992; 29 : $65-68$. 